Mathematical Research Letters 11, 629-647 (2004)

\title{
$K$-THEORY ASSOCIATED TO VERTEX OPERATOR ALGEBRAS
}

\author{
Chongying Dong, Kefeng Liu, Xiaonan Ma, and Jian Zhou
}

\begin{abstract}
We introduce two $K$-theories, one for vector bundles whose fibers are modules of vertex operator algebras, another for vector bundles whose fibers are modules of associative algebras. We verify the cohomological properties of these $K$-theories, and construct a natural homomorphism from the VOA $K$-theory to the associative algebra $K$-theory.
\end{abstract}

\section{Introduction}

Since its introduction by Grothendieck, Atiyah and Hirzebruch, $K$-theory has found many applications in algebraic geometry, topology and differential geometry. $K$-theories in different settings lead to the Grothendieck-HirzebruchRiemann-Roch theorem and the Atiyah-Singer index theory. Originally such theory was developed starting from vector bundles. Note that one can regard vector spaces, which are fibers of vector bundles, simply as $\mathbb{C}$-modules. It is natural to consider bundles of modules over other algebras.

The theory of vertex operator algebras has been very much developed in the last eighteen years. Playing important roles in the study of elliptic genus and Witten genus, the highest weight representations of Heisenberg and affine Kac-Moody algebras provide important examples of vertex operator algebras. In this note we introduce a $K$-theory for vector bundles of modules of vertex operator algebras, and a $K$-theory for vector bundles of modules of associative algebras. We verify the cohomology theory properties of these $K$-theories - the exact sequences. We also give a natural homomorphism from the vertex operator algebra $K$-group to the associative algebra $K$-group when the associative algebra is the Zhu's algebra of the vertex operator algebra.

We follow closely the construction of topological $K$-theory by Atiyah in [1] to verify the cohomological properties of the two new $K$-groups. We need certain deep property of vertex operator algebras, such as the existence of the nondegenerate symmetric bilinear form, to get exact sequences. Our original motivation

Received May 5, 2004.

First author partially supported by NSF grants and a research grant from the Committee on Research, UC Santa Cruz.

Second author partially supported by an NSF grant. 
for this work is to understand elliptic cohomology which only has homotopytheoretical construction. We plan to study the applications of such $K$-theories, in particular their relationship to the elliptic genus and elliptic cohomology.

\section{Vertex operator algebras and modules}

We recall the definitions of vertex operator algebras and their modules (cf. $[2],[4],[9],[8],[15])$ in this section. Rational vertex operator algebras and contragredient modules are discussed. We will also introduce the category $\mathcal{O}_{V}$ of ordinary $V$-modules for the purpose of $K$-theory developed in this paper. The Heisenberg vertex operator algebras and vertex operator algebras associated to the highest weight representations for affine Kac-Moody algebras are reviewed. They have played important roles in the study of elliptic genus and Witten genus in the literature (cf.[14]).

2.1. Vertex operator algebras and modules. Let $z, z_{0}, z_{1}, z_{2}$ be commuting formal variables. We shall use the formal power series $\delta(z)=\sum_{n \in \mathbb{Z}} z^{n}$ which is formally the expansion of the $\delta$-function at $z=1$. For the properties of the $\delta$-function see e.g. [9], [8] and [3].

A vertex operator algebra is a $\mathbb{Z}$-graded vector space:

$$
V=\bigoplus_{n \in \mathbb{Z}} V_{n} ; \quad \text { for } \quad v \in V_{n}, \quad n=\text { wt } v
$$

such that $\operatorname{dim} V_{n}<\infty$ for all $n \in \mathbb{Z}$ and $V_{n}=0$ if $n$ is sufficiently small; equipped with a linear map

$$
\begin{aligned}
& V \rightarrow(\text { End } V)\left[\left[z, z^{-1}\right]\right] \\
& v \mapsto Y(v, z)=\sum_{n \in \mathbb{Z}} v_{n} z^{-n-1} \quad\left(v_{n} \in \text { End } V\right)
\end{aligned}
$$

and with two distinguished vectors $1 \in V_{0}, \omega \in V_{2}$ satisfying the following conditions for $u, v \in V$ :

$$
\begin{aligned}
& u_{n} v=0 \quad \text { for } n \text { sufficiently large; } \\
& Y(\mathbf{1}, z)=1 ; \\
& Y(v, z) \mathbf{1} \in V[[z]] \text { and } \lim _{z \rightarrow 0} Y(v, z) \mathbf{1}=v ;
\end{aligned}
$$

and there exists a nonnegative integer $n$ depending on $u, v$ such that

$$
\begin{gathered}
\left(z_{1}-z_{2}\right)^{n}\left[Y\left(u, z_{1}\right), Y\left(v, z_{2}\right)\right]=0 ; \\
{[L(m), L(n)]=(m-n) L(m+n)+\frac{1}{12}\left(m^{3}-m\right) \delta_{m+n, 0}(\operatorname{rank} V),}
\end{gathered}
$$

for $m, n \in \mathbb{Z}$, and

$$
\begin{aligned}
& L(0) v=n v=(\text { wt } v) v \quad \text { for } \quad v \in V_{n}(n \in \mathbb{Z}), \\
& \frac{d}{d z} Y(v, z)=[L(-1), Y(u, z)]=Y(L(-1) v, z)
\end{aligned}
$$


where

$$
L(n)=\omega_{n+1} \quad \text { for } \quad n \in \mathbb{Z}, \quad \text { i.e., } \quad Y(\omega, z)=\sum_{n \in \mathbb{Z}} L(n) z^{-n-2} .
$$

This completes the definition. We denote the vertex operator algebra just defined by $(V, Y, \mathbf{1}, \omega)$ (or briefly, by $V)$. The series $Y(v, z)$ are called vertex operators.

Remark 2.1. Any commutative associative algebra $V$ over $\mathbb{C}$ is a vertex operator algebra with $\mathbf{1}=1, \omega=0$ and $Y(u, z) v=u v$ for $u, v \in V$. In particular, $\mathbb{C}$ itself is a vertex operator algebra.

An automorphism $g$ of the vertex operator algebra $V$ is a linear automorphism of $V$ preserving 1 and $\omega$ such that the actions of $g$ and $Y(v, z)$ on $V$ are compatible in the sense that $g Y(v, z) g^{-1}=Y(g v, z)$ for $v \in V$. Then $g V_{n} \subset V_{n}$ for $n \in \mathbb{Z}$. The group of all automorphisms of the vertex operator algebra $V$ is denoted by $\operatorname{Aut}(V)$.

We now define admissible modules and ordinary modules for vertex operator algebras. An admissible $V$-module

$$
M=\bigoplus_{n=0}^{\infty} M(n)
$$

is a $\mathbb{Z}$-graded vector space equipped with a linear map

$$
\begin{aligned}
& V \longrightarrow(\text { End } M)\left[\left[z, z^{-1}\right]\right] \\
& v \longmapsto Y_{M}(v, z)=\sum_{n \in \mathbb{Z}} v_{n} z^{-n-1} \quad\left(v_{n} \in \text { End } M\right)
\end{aligned}
$$

which satisfies the following conditions; for $u, v \in V, w \in M, n \in \mathbb{Z}$,

$$
\begin{aligned}
& u_{n} w=0 \text { for } n \gg 0, \\
& Y_{M}(\mathbf{1}, z)=1
\end{aligned}
$$

$$
\begin{gathered}
z_{0}^{-1} \delta\left(\frac{z_{1}-z_{2}}{z_{0}}\right) Y_{M}\left(u, z_{1}\right) Y_{M}\left(v, z_{2}\right)-z_{0}^{-1} \delta\left(\frac{z_{2}-z_{1}}{-z_{0}}\right) Y_{M}\left(v, z_{2}\right) Y_{M}\left(u, z_{1}\right) \\
=z_{2}^{-1} \delta\left(\frac{z_{1}-z_{0}}{z_{2}}\right) Y_{M}\left(Y\left(u, z_{0}\right) v, z_{2}\right),
\end{gathered}
$$

which is called the Jacobi identity. Here all binomial expressions $\left(z_{i}-z_{j}\right)^{n}$ are to be expanded in nonnegative integral powers of second variable $z_{j}$. This identity is interpreted algebraically as follows: if this identity is applied to a single vector of $M$ then the coefficient of each monomial in $z_{0}, z_{1}, z_{2}$ is a finite sum in $M$;

$$
u_{m} M(n) \subset M(\text { wt } u-m-1+n)
$$

if $u$ is homogeneous. We denote the admissible $V$-module by $M=\left(M, Y_{M}\right)$.

Homomorphisms and isomorphisms of admissible modules are defined as expected. It is easy to see that a grading shift of $M$ does not change the module structure. So we will assume that the top level $M(0)$ of $M$ is nonzero. 
Remark 2.2. Let $\left(M, Y_{M}\right)$ be an admissible $V$-module. Then $L(-1)$-derivation property

$$
Y_{M}(L(-1) v, z)=\frac{d}{d z} Y_{M}(v, z)
$$

holds. Moreover, the component operators of $Y_{M}(\omega, z)$ generate a copy of the Virasoro algebra of central charge rank $V$ (see [4]).

Definition 2.3. Let $G$ be a subgroup of $\operatorname{Aut}(V)$ and $M=\left(M, Y_{M}\right)$ an admissible $V$-module. We say that $G$ acts on $M$ as automorphisms if $M$ is a $G$-module and $g Y_{M}(v, z) g^{-1}=Y_{M}(g v, z)$ on $M$ for all $g \in G, v \in V$.

Remark 2.4. In general $G$ does not act on an admissible module. Assume that $V=\sum_{n=0}^{\infty} V_{n}$ with $V_{0}=\mathbb{C} 1$. Then $V_{1}$ is a Lie algebra. Let $N$ be the subgroup of $\operatorname{Aut}(V)$ generated by $e^{u_{0}}$ for $u \in V_{1}$. The group $N$ acts on any admissible module.

An (ordinary) $V$-module is an admissible $V$-module $M$ which carries a $\mathbb{C}$ grading induced by the spectrum of $L(0)$. That is, we have

$$
M=\bigoplus_{\lambda \in \mathbb{C}} M_{\lambda}
$$

where $M_{\lambda}=\{w \in M \mid L(0) w=\lambda w\}$. Moreover we require that $\operatorname{dim} M_{\lambda}$ is finite and for fixed $\lambda, M_{n+\lambda}=0$ for all small enough integers $n$. Let $P(M)=\{\lambda \in$ $\left.\mathbb{C} \mid M_{\lambda} \neq 0\right\}$. An element $\lambda \in P(M)$ is called a weight of $M$.

Definition 2.5. A vertex operator algebra $V$ is called rational if any admissible $V$-module is a direct sum of irreducible admissible $V$-modules.

It is proved in [5, Theorem 8.1] (also see [15]) that if $V$ is a rational vertex operator algebra then every irreducible admissible $V$-module is an ordinary $V$-module and $V$ has only finitely many irreducible admissible modules up to isomorphism.

Definition 2.6. For each $\lambda \in \mathbb{C}$ we set $D(\lambda)=\{\lambda+n \mid 0 \leq n \in \mathbb{Z}\}$. We define a category $\mathcal{O}_{V}$ of ordinary $V$-modules such that for each $M \in \mathcal{O}_{V}$ there are finitely many complex numbers $\lambda_{1}, \ldots, \lambda_{s}$ such that $P(M) \subset \cup_{i=1}^{s} D\left(\lambda_{i}\right)$.

Note that if $M$ is an irreducible $V$-module then there exists $\lambda \in \mathbb{C}$ such that $M=\bigoplus_{n>0} M_{\lambda+n}$. Thus any irreducible $V$-module lies in the category $\mathcal{O}_{V}$.

The definition of category $\mathcal{O}_{V}$ is very similar to the category $\mathcal{O}$ in the representation theory for Kac-Moody algebras (cf. [12]). The purpose for such definition will be clear from the discussion in the next section.

Remark 2.7. (1) If $V$ is rational then the category $\mathcal{O}_{V}$ is exactly the category of ordinary $V$-modules.

(2) If $U$ and $V$ are two vertex operator algebras, there is a functor from $\mathcal{O}_{U} \times \mathcal{O}_{V} \rightarrow \mathcal{O}_{U \otimes V}$ such that $(M, N)$ is mapped to $M \otimes N$. 
Next we recall the notion of contragredient module from [8]. Let $M=$ $\bigoplus_{\lambda \in \mathbb{C}} M_{\lambda}$ be a $V$-module and $M^{\prime}=\bigoplus_{\lambda \in \mathbb{C}} M_{\lambda}^{*}$ the graded dual. We denote the natural pairing on $M^{\prime} \times M$ by $\left(w^{\prime}, w\right)$ for $w^{\prime} \in M^{\prime}$ and $w \in M$. Then $\left(M^{\prime}, Y\right)$ is also a $V$-module such that

$$
\left(Y(v, z) w^{\prime}, w\right)=\left(w^{\prime}, Y\left(e^{z L(1)}\left(-z^{-2}\right)^{L(0)} v, z^{-1}\right) w\right)
$$

for $v \in V, w^{\prime} \in M^{\prime}$ and $w \in W$ (see [8, Theorem 5.2.1]). Moreover, $M^{\prime}$ is irreducible if and only if $M$ is irreducible.

Let $W$ be a $V$-module. A bilinear form on $W$ is called invariant if

$$
\left(Y(v, z) w_{1}, w_{2}\right)=\left(w_{1}, Y\left(e^{z L(1)}\left(-z^{-2}\right)^{L(0)} v, z^{-1}\right) w_{2}\right)
$$

for $w_{i} \in W$ and $v \in V$.

Lemma 2.8. For any $V$-module $M, M \oplus M^{\prime}$ has a natural nondegenerate symmetric invariant bilinear form defined by

$$
\left(u+u^{\prime}, w+w^{\prime}\right)=\left(u, w^{\prime}\right)+\left(w, u^{\prime}\right)
$$

for any $u, w \in M$ and $u^{\prime}, w^{\prime} \in M^{\prime}$. In particular, any $V$-module can be embedded into a module with a nondegenerate symmetric invariant bilinear form.

2.2. Examples. In order to discuss examples of vertex operator algebra bundles in the next few sections we recall some well-known vertex operator algebras.

(a) Heisenberg vertex operator algebra $M(1)$ of dimension $d$ (cf. [9]). Let $\mathfrak{h}$ be complex vector space of dimension $d$ with a nondegenerate symmetric bilinear from $($,$) . Viewing \mathfrak{h}$ as an abelian Lie algebra, we consider the corresponding affine Lie algebra $\hat{\mathfrak{h}}=\mathfrak{h} \otimes \mathbb{C}\left[t, t^{-1}\right] \oplus \mathbb{C} K$ with bracket

$$
\begin{gathered}
{\left[x \otimes t^{m}, y \otimes t^{n}\right]=\delta_{m+n, 0}(x, y) K,} \\
{[K, \hat{\mathfrak{h}}]=0,}
\end{gathered}
$$

where $x(m)=x \otimes t^{m}$ for $x \in \mathfrak{h}$ and $m \in \mathbb{Z}$. Form the induced module

$$
M(1)=U(\hat{\mathfrak{h}}) \otimes_{U(\mathfrak{h} \otimes \mathbb{C}[t] \oplus \mathbb{C} K)} \mathbb{C},
$$

where $\mathfrak{h} \otimes \mathbb{C}[t]$ acts trivially on $\mathbb{C}$ and $K$ acts as 1 . Let $\left\{\alpha_{1}, \ldots, \alpha_{d}\right\}$ be an orthonormal basis of $\mathfrak{h}$. Then $M(1)$ is isomorphic linearly to the symmetric algebra

$$
S\left(\mathfrak{h} \otimes t^{-1} \mathbb{C}\left[t^{-1}\right]\right)=\mathbb{C}\left[\alpha_{i}(-n) \mid i=1, \ldots, d, n>0\right] .
$$

Set $\mathbf{1}=1$ and $\omega=\frac{1}{2} \sum_{i=1}^{d} \alpha_{i}(-1)^{2}$. Then $M(1)$ is the Heisenberg vertex operator algebra with vacuum 1 and Virasoro element $\omega$ (cf. [9, Chapter 8]). Let $O(\mathfrak{h})$ be the orthogonal group of $\mathfrak{h}$. The automorphism group of $M(1)$ is exactly $O(\mathfrak{h})$ (cf. [6, Proposition 2.3]).

(b) Vertex operator algebra associated to the highest weight modules for affine Kac-Moody algebras (cf. [3], [10], [13]). Let $\mathfrak{g}=\mathfrak{h} \oplus \oplus_{\alpha \in \Delta} \mathfrak{g}_{\alpha}$ be a finite dimensional simple Lie algebra with a Cartan subalgebra $\mathfrak{h}$ and the corresponding root 
system $\Delta$. We fix the positive roots $\Delta_{+}$and assume that $\theta$ is the longest positive root. Denote $P_{+}$the set of dominant weights. Let $(\cdot, \cdot)$ be a nondegenerate symmetric invariant bilinear form on $\mathfrak{g}$ such that $(\theta, \theta)=2$.

The affine Kac-Moody algebra is

$$
\hat{\mathfrak{g}}=\mathfrak{g} \otimes \mathbb{C}\left[t, t^{-1}\right] \oplus \mathbb{C} K,
$$

with bracket

$$
\begin{gathered}
{[a(m), b(n)]=[a, b](m+n)+m \delta_{m+n, 0}(a, b) K,} \\
{[K, \hat{\mathfrak{g}}]=0,}
\end{gathered}
$$

where $a(m)=a \otimes t^{m}$ for $a \in \mathfrak{g}$ and $m \in \mathbb{Z}$. If $M$ is an irreducible $\hat{\mathfrak{g}}$-module then the center $K$ acts as a constant $k$ which is called the level of the module.

Let $M$ be a $\mathfrak{g}$-module and $k$ a complex number. The generalized Verma modules of level $k$ associated to $M$ is

$$
\widehat{M}_{k}=U(\hat{\mathfrak{g}}) \otimes_{U(\mathfrak{g} \otimes \mathbb{C}[t] \oplus \mathbb{C} K)} M
$$

where $\mathfrak{g} \otimes t \mathbb{C}[t] \cdot M=0, \mathfrak{g} \otimes t^{0}$ acts on $M$ as $\mathfrak{g}$ and $K=k$ on $M$.

Let $L(\lambda)$ be the highest weight module for $\mathfrak{g}$ with highest weight $\lambda \in \mathfrak{h}$. Set $V(k, \lambda)=\widehat{L(\lambda)}_{k}$ and denote $L(k, \lambda)$ the unique irreducible quotient. Then $L(k, \lambda)$ is integrable or unitary if and only if $k$ is a nonnegative integer, $\lambda \in P_{+}$, and $(\lambda, \theta) \leq k$.

Denote the dual Coxeter number of $\mathfrak{g}$ by $h^{\vee}$. Then $h^{\vee}$ can be defined by $\sum_{\alpha \in \Delta} \frac{(\alpha, \alpha)}{2}=d h^{\vee}$ where $d$ is the rank of $\mathfrak{g}$. Then we have

(a) Any $\hat{\mathfrak{g}}$-quotient module of $V(k, 0)$ is a vertex operator algebra if $k+h^{\vee} \neq 0$.

(b) $L(k, 0)$ is rational if and only if $k$ is a nonnegative integer. In this case, the irreducible $L(k, 0)$-modules are exactly the level $k$ unitary highest weight modules. In particular, if $\mathfrak{g}$ is the Lie algebra of type $D_{d}$, then $\mathfrak{g}$ is the affine Lie algebra $D_{d}^{(1)}$ which has exactly 4 level 1 unitary highest weight modules. These modules are used in the construction of elliptic genera.

The automorphism groups of $V(k, 0)$ and $L(k, 0)$ are exactly the automorphism group of the Lie algebra $\mathfrak{g}$ (cf. [6]).

\section{Vertex operator algebras bundles}

In this section we use the category $\mathcal{O}_{V}$ to define vertex operator algebra bundles over a compact space. This generalizes the notion of complex vector bundles. The main result in this section is that for any $V$-bundle $E$ there is another $V$-bundle $F$ such that $E \oplus F$ is a trivial bundle. As in the classical case, this result is crucial in defining VOA $K$-groups and studying the cohomology properties of the $K$-groups. We also prove that a $V$-bundle is essentially a vector bundle if $V$ is rational. 
3.1. Definition of a VOA bundles and some consequences. We now fix a vertex operator algebra $V$. Let $X$ be a compact topological space.

Definition 3.1. Let $M \in \mathcal{O}_{V}$. A $V$-bundle $E$ over $X$ with fiber $M$ is a direct sum of vector bundles $E=\oplus_{\lambda \in \mathbb{C}} E^{\lambda}$ over $X$ such that all transition functions are $V$-module isomorphisms. That is, there exists an open covering $\left\{X_{\alpha}\right\}_{\alpha \in I}$ of $X$ and a family of continuous isomorphism of vector bundles $H_{\alpha}=\left(H_{\alpha}^{\lambda}\right.$ : $\left.\left.E^{\lambda}\right|_{X_{\alpha}} \rightarrow X_{\alpha} \times M_{\lambda}\right)_{\lambda \in \mathbb{C}}$ with $M=\oplus_{\lambda \in \mathbb{C}} M_{\lambda}$ a $V$-module such that if we denote by $\left(H_{\alpha}^{\lambda} \circ\left(H_{\beta}^{\lambda}\right)^{-1}\right)_{\lambda \in \mathbb{C}}=\left(g_{\alpha \beta}^{\lambda}\right)_{\lambda \in \mathbb{C}}$, then $g_{\alpha \beta}(x)=\left(g_{\alpha \beta}^{\lambda}(x)\right)_{\lambda \in \mathbb{C}}: M \rightarrow M$ is a $V$-module isomorphism for any $x \in X_{\alpha} \cap X_{\beta}$.

Remark 3.2. (1) If $V=\mathbb{C}$ as in Remarks 2.1, the $V$-bundle defined here is exactly the classical complex vector bundle over $X$.

(2) Let $U$ and $V$ be two vertex operator algebras and $E, F$ be the $U$ and $V$-bundles over $X$ respectively. Then $E \otimes F$ is a $U \otimes V$-bundle over $X$ (see Remark 2.7). In particular, If $U=\mathbb{C}$ then $E \otimes F$ again is a $V$-bundle over $X$.

(3) One could use the full module category of $V$-modules to define a $V$ bundle but the property (2) in this remark would not be true. But this property is fundamental if one wants to construct a ring instead of a group from vertex operator algebra bundles (see Corollary 4.3).

(4) One can give a different definition of $V$-bundle using a subgroup $G$ of $\operatorname{Aut}(V)$. In this case, we denote $\mathcal{O}_{V, G}$ the subcategory of $\mathcal{O}_{V}$ consisting of $V$ modules $M$ such that $G$ acts on $M$ as automorphisms. The transition functions $g_{\alpha, \beta}(x)$ now are required to be in $G$. Most results in this paper hold for this definition of $V$-bundles.

(5) There was a notion of vertex operator algebra bundle given in [7]. But our definition is different from theirs.

Sub-bundles and quotient bundles, direct sum of bundles are defined as expected. Let $E, F$ be two $V$-bundles on $X$, a map $f: E \rightarrow F$ is called a $V$-bundle homomorphism if there exist a family of continuous homomorphisms of vector bundles $f^{\lambda}: E^{\lambda} \rightarrow F^{\lambda}$ such that $f=\left(f^{\lambda}\right)_{\lambda \in \mathbb{C}}: E \rightarrow F$ is a $V$-module homomorphism for any $x \in X$. We call a $V$-bundle homomorphism $f: E \rightarrow F$ an epimorphism, monomorphism and isomorphism of $V$-bundles if $f^{\lambda}$ is so for any $\lambda \in \mathbb{C}$.

We say a $V$-bundle $E$ is trivial if there exists a $V$-bundle isomorphism $\varphi$ : $E \rightarrow X \times M$, here $X \times M$ is the natural $V$-bundle on $X$ with the $V$-module $M$ as fibers.

We now discuss some well known examples of vertex operator algebra bundles which have been used in the literature to study elliptic genus and Witten genus.

(a) If $X$ is an oriented Riemannian manifold, then the transition functions of the complexified tangent bundle $T_{\mathbb{C}} X$ lie in the special orthogonal group $S O(d)$ where $d$ is the dimension of $X$. Then $\otimes_{n>0} S_{q^{n}}\left(T_{\mathbb{C}} X\right)$ is a $M(1)^{S O(d)}$-bundle. Here and below, $S_{t}(E)=1+t E+t^{2} \operatorname{Sym}^{2}(E)+\cdots, \Lambda_{t}(E)=1+t E+t^{2} \Lambda^{2}(E)+\cdots$ are respectively the symmetric and wedge operation of a complex vector bundle E. $M(1)$ is the Heisenberg vertex operator algebra of dimension $d$ with $S O(d)$ 
as a subgroup of $\operatorname{Aut}(M(1))$ and $M(1)^{S O(d)}$ is the $S O(d)$-invariants of $M(1)$ which is a vertex operator subalgebra of $M(1)$. Similarly, $\otimes_{n \geq 0} \Lambda_{q^{n+1 / 2}}\left(T_{\mathbb{C}} X\right)$ is an $L(1,0)^{S O(d)}$-bundle where $L(1,0)$ is the level one module for the affine algebra $D_{d / 2}^{(1)}$. Here we assume that $d$ is even in this case.

(b) If $X$ is further assumed to be a spin manifold, we denote the spin bundle by $S$. Then $S \otimes \otimes_{n>0} \Lambda_{q^{n}}\left(T_{\mathbb{C}} X\right)$ is also a $L(1,0)^{S O(d)}$-bundle.

Let $E$ be a $V$-bundle over $X$. Set $E^{\prime}=\oplus_{\lambda \in \mathbb{C}}\left(E^{\lambda}\right)^{*}$. Then $E^{\prime}$ is also a $V$-bundle in an obvious way.

Lemma 3.3. Let $E$ be a $V$-bundle on $X$. Then

(1) $E \oplus E^{\prime}$ is a $V$-bundle with a nondegenerate symmetric invariant bilinear form induced from the natural bilinear from on $M \oplus M^{\prime}$. In particular, any $V$ bundle on $X$ can be embedded into a bundle with a nondegenerate symmetric invariant bilinear form.

(2) Let $\left\{g_{\alpha \beta}^{*}\right\}$ be the transition functions of $E^{\prime}$. Then we have

$$
\left(g_{\alpha \beta}^{*}(x) s^{*}, g_{\alpha \beta}(x) s\right)=\left(s^{*}, s\right)
$$

for any $\alpha, \beta \in I, x \in X_{\alpha} \cap X_{\beta}$ and $s \in M, s^{*} \in M^{\prime}$.

Proof : (1) follows from Lemma 2.8 and (2) is clear.

Proposition 3.4. For any $V$-bundle $E$ there exists another $V$-bundle $F$ such that $E \oplus F$ is a trivial $V$-bundle.

Proof : By Lemma 3.3 we can assume that there is a nondegenerate invariant symmetric bilinear form on $E$.

Certainly we can take a finite covering $\left\{X_{\alpha}\right\}_{\alpha \in I}$ in the Definition 3.1. Write $H_{\alpha}=\left(\pi, h_{\alpha}\right)$ for $\alpha \in I$ where $\pi: E \rightarrow X$ is the natural projection map and $h_{\alpha}(e) \in M$. Let $\left\{p_{\alpha}: X \rightarrow \mathbb{R}_{+}\right\}_{\alpha \in I}$ be a family of continuous functions on $X$ such that $\left\{p_{\alpha}^{2}\right\}_{\alpha \in I}$ is a partition of unity on $X$ associated to the covering $\left\{X_{\alpha}\right\}_{\alpha \in I}$. Define a $V$-bundle homomorphism

$$
\sigma: E \rightarrow X \times M^{\oplus n}
$$

by $\sigma(e)=\left(x,\left(p_{\alpha}(x) h_{\alpha}(e)\right)_{\alpha \in I}\right)$ where $x=\pi(e)$ and $n$ is the cardinality of $I$. Since $p_{\alpha}(x)>0$ for some $\alpha \in I$, we see that $\sigma$ is a monomorphism. So we can identify $E$ with its image $\sigma(E)$ in the trivial $V$-bundle $X \times M^{\oplus n}$.

By extending the bilinear form from $M$ to $M^{\oplus n}$ componentwisely, we have a nondegenerate invariant symmetric bilinear form on $X \times M^{\oplus n}$. By Lemma 3.3, 
the transition functions preserve the bilinear form on $M$, thus for any $e, f \in E_{x}$

$$
\begin{gathered}
(\sigma(e), \sigma(f))_{x}=\sum_{\alpha \in I}\left(p_{\alpha}(x) h_{\alpha}(e), p_{\alpha}(x) h_{\alpha}(f)\right) \\
=\sum_{\alpha \in I}\left(g_{\alpha \beta}(x) h_{\beta}(e), g_{\alpha \beta}(x) h_{\beta}(f)\right) p_{\alpha}^{2}(x) \\
=\left(h_{\beta}(e), h_{\beta}(f)\right) \sum_{\alpha \in I} p_{\alpha}^{2}(x) \\
=(e, f)_{x},
\end{gathered}
$$

where $\beta \in I$ is fixed such that $x \in X_{\beta}$. Thus the homomorphism $\sigma$ preserves the bilinear form and the restriction of the bilinear form to $\sigma(E)$ is nondegenerate. Set $F=\sigma(E)^{\perp}$. Then $F$ is still a $V$-bundle on $X$ and $X \times M^{\oplus n}=\sigma(E) \oplus F \cong$ $E \oplus F$, as desired.

3.2. The structure of $V$-bundles for rational VOA $V$. Next we discuss a special case when $V$ is a rational vertex operator algebra. Then $V$ has only finitely many irreducible modules up to isomorphisms, say $\left\{M^{1}, \ldots, M^{p}\right\}$. Any $V$-module $W$ is isomorphic to $\oplus_{i=1}^{p} n_{i} M^{i}$. The nonnegative integer $n_{i}$ is called the index of $M^{i}$ in $W$ and is denoted by $\left[W: M^{i}\right]$. Given another $V$-module $M$ we define the Grassmannian variety $G(W, M)$ of $W$ to be the set of $V$-submodules of $W$ isomorphic to $M$. It is clear that if $\left[M: M^{i}\right]>\left[W: M^{i}\right]$ for some $i, G(W, M)$ is an empty set. So we restrict ourselves to the case when $M$ is a submodule of $W$. Let $W=\bigoplus_{i=1}^{p} k_{i} M^{i}$ with $k_{i} \leq n_{i}$. Then

$$
G(W, M) \cong G\left(n_{1}, k_{1}\right) \times \cdots \times G\left(n_{p}, k_{p}\right)
$$

as varieties where $G(n, k)$ is the classical Grassmann variety. That is $G(n, k)$ is the set of $k$-dimensional subspaces of an $n$-dimensional complex vector space. Clearly, $G(W, M)$ is compact as each $G\left(n_{i}, k_{i}\right)$ is compact.

Note that over $G(W, M)$ there is a canonical $V$-bundle $E(W, M)$ whose total space consists of $(H, x)$ where $H$ is a $V$-submodule of $W$ isomorphic to $M$ and $x \in H$.

Proposition 3.5. Let $E$ be a $V$-bundle over $X$ whose fiber is isomorphic to a $V$-module $M$. Then there is a bundle homomorphism $f$ from $E$ to $E\left(M^{\oplus n}, M\right)$ such that $E$ is isomorphic to $f^{*} E\left(M^{\oplus n}, M\right)$.

Proof : Recall that $\left\{X_{\alpha} \mid \alpha \in I\right\}$ is an open covering of $X$ and $\left\{p_{\alpha}^{2}\right\}_{\alpha \in I}$ is a partition of the unity with $p_{\alpha}$ being supported by $X_{\alpha}$. Also recall the isomorphism

$$
H_{\alpha}:\left.E\right|_{X_{\alpha}} \rightarrow X_{\alpha} \times M
$$

which sends $e$ to $\left(\pi(e), h_{\alpha}(e)\right)$.

In order to construct the bundle homomorphism $f$ it is enough to define a $V$ module monomorphism $\bar{f}: E_{x} \rightarrow M^{\oplus n}$. Let $e \in E_{x}$. As in the classical case, we set $\bar{f}(e)=\left(p_{\alpha}(e) h_{\alpha}(e)\right)_{\alpha \in I} \in M^{\oplus n}$. It is clear $\bar{f}$ is a $V$-module monomorphism. As usual, $f(e)=\left(\bar{f}\left(E_{x}\right), \bar{f}(e)\right)$. Then $f$ is a bundle homomorphism from $E$ to 
$E\left(M^{\oplus n}, M\right)$ such that $E$ is isomorphic to $f^{*} E\left(M^{\oplus n}, M\right)$.

The result in Proposition 3.5 is not surprising. Such a result is well known for ordinary vector bundles.

Next we show that if $V$ is rational, any $V$-bundle is determined by certain vector bundles. Let $E$ be a $V$-bundle with fiber $M$. Let $M=\oplus_{i=1}^{p} W_{i} \otimes M^{i}$ where as before $\left\{M^{1}, \ldots, M^{p}\right\}$ is a complete list of inequivalent irreducible $V$-modules and $W_{i}$ is the space of multiplicity of $M^{i}$ in $M$. Then each $g_{\alpha \beta}$ defines a map $h_{\alpha \beta}: X_{\alpha} \cap X_{\beta} \rightarrow \bigoplus_{i=1}^{p} \operatorname{End}\left(W_{i}\right)$. For each $i$ we define a vector bundle $V(E)^{i}$ over $X$ with fiber $W_{i}$ and transition functions $\left\{h_{\alpha \beta} \mid \alpha, \beta \in I\right\}$. For any $V$-module $N \in \mathcal{O}_{V}$ we denote the trivial $V$-bundle on $X$ by $\underline{N}$. That is, $\underline{N}=X \times N$. From Remark 3.2, we have a $V$-bundle $V(E)^{i} \otimes \underline{M}^{i}$. So we have proved the following proposition.

Proposition 3.6. If $V$ is rational then for any $V$-bundle $E$ over $X$ there are vector bundles $V(E)^{i}$ for $i=1, \ldots, p$ such that $E$ is isomorphic to $\oplus_{i=1}^{p} V(E)^{i} \otimes$ $\underline{M}^{i}$.

\section{Definition of VOA $K$-group}

In this section we define a $K$-group associated to $V$-bundles for a vertex operator algebra $V$. We follow closely the set-up of [1] with certain necessary extensions.

4.1. Definition of $K_{V}(X)$. Let $X$ be a compact space, we denote by $\mathcal{V}_{V}(X)$ the set of isomorphic classes of $V$-bundles over $X . \mathcal{V}_{V}(X)$ is an abelian semigroup with addition given by the direct sum. We also denote by $K_{V}(X)$ the abelian group generated by the equivalence classes of $V$-bundle $[E]$. Then the elements of $K_{V}(X)$ are of the form $[E]-[F]$.

Remark 4.1. If $V=\mathbb{C}$, the $K_{V}(X)$ is the classical $K(X)$ as defined in [1].

First from Remark 3.2 we have the following:

Lemma 4.2. If $U, V$ are two vertex operator algebras then the tensor product of the bundles induces a natural group homomorphism $K_{U}(X) \otimes_{\mathbb{Z}} K_{V}(X) \rightarrow$ $K_{U \otimes V}(X)$. In particular, if $U=\mathbb{C}$, then $K_{V}(X)$ is a natural $K(X)$-module (cf. Remark 4.1).

The following corollary is an immediate consequence of Lemma 4.2.

Corollary 4.3. For any vertex operator algebra $V$,

$$
\oplus_{n \geq 0} K_{V \otimes n}(X)
$$

is a commutative algebra over $K(X)$ where $V^{\otimes 0}$ is understood to be $\mathbb{C}$.

The following proposition is crucial to prove the cohomological properties of $K_{V}(X)$. We follow the argument of [11, Proposition 1.7] which avoids the use of the extension of the section of $\operatorname{Aut}_{V}(E)$ from a compact subset to a open neighborhood, as $\operatorname{Aut}_{V}(E)$ may be infinite dimensional. 
Lemma 4.4. Let $Y$ be a compact Hausdorff space, $f_{t}: Y \rightarrow X(0 \leq t \leq 1) a$ homotopy and $E$ a $V$-bundle over $X$. Then

$$
f_{0}^{*} E \simeq f_{1}^{*} E .
$$

Proof : Denote by $I$ the unit interval and let $f: Y \times I \rightarrow X$ be the homotopy, so that $f(y, t)=f_{t}(y)$, and let $\pi: Y \times I \rightarrow Y$ denote the projection onto the first factor.

At first, we can choose a finite open covering $\left\{Y_{x_{i}}\right\}_{i=1}^{n}$ of $Y$ so that $f^{*} E$ is trivial over each $Y_{x_{i}} \times I$. In fact, for each $x \in Y$ we can find open neighborhood $U_{x, 1}, \cdots, U_{x, k}$ in $Y$ and a partition $0=t_{0}<t_{1} \cdots<t_{k}=1$ of $[0,1]$ such that the bundle is trivial over each $U_{x, i} \times\left[t_{i-1}, t_{i}\right]$. Set $Y_{x}=U_{x, 1} \cap \cdots \cap U_{x, k}$. Now we claim that $f^{*} E$ is trivial over $Y_{x} \times I$. To see this, let $h_{i}:\left.f^{*} E\right|_{Y_{x} \times\left[t_{i-1}, t_{i}\right]} \rightarrow$ $Y_{x} \times\left[t_{i-1}, t_{i}\right] \times M$ be the trivializing isomorphisms. We take the $V$-bundle isomorphism $h_{2}^{\prime}(y, t)=\left(h_{0} \circ h_{1}^{-1}\right)\left(y, t_{1}\right) \circ h_{1}(y, t):\left.f^{*} E\right|_{Y_{x} \times\left[t_{1}, t_{2}\right]} \rightarrow Y_{x} \times\left[t_{1}, t_{2}\right] \times$ $M$, then $h_{1}=h_{2}^{\prime}$ on $Y_{x} \times\left\{t_{1}\right\}$, thus they define a trivialization on $Y_{x} \times\left[t_{0}, t_{2}\right]$, and in this way, we know that $f^{*} E$ is trivial over $Y_{x} \times I$. Now, as $Y$ is compact, there exist $\left\{Y_{x_{i}}\right\}_{i=1}^{n}$ which cover $Y$.

Let $p_{i}$ be a partition of unity of $Y$ with support of $p_{i}$ contained in $Y_{x_{i}}$. For $i \geq 0$, set $q_{j}=\sum_{i=1}^{j} p_{i}$. In particular $q_{0}=0$ and $q_{n}=1$. Let $W_{i}$ be the graph of $q_{i}$, the subspace of $Y \times I$ consisting of points of the form $\left(x, q_{i}(x)\right)$, and let $\pi_{i}: E_{i} \rightarrow W_{i}$ be the restriction of the bundle $E$ over $W_{i}$. Since $E$ is trivial on $Y_{x_{i}} \times I$, the natural projection homeomorphism $W_{i} \rightarrow W_{i-1}$ lifts to a homeomorphism $g_{i}: E_{i} \rightarrow E_{i-1}$ which is identity outside $\pi_{i}\left(Y_{x_{i}}\right)$ and which takes each fiber of $E_{i}$ isomorphically onto the corresponding fiber of $E_{i-1}$. The composition $g=g_{1} \circ g_{2} \cdots \circ g_{n}$ is then an isomorphism from the restriction of $E$ over $Y \times\{1\}$ to the restriction on $Y \times\{0\}$.

Lemma 4.5. (1) Any element of $K_{V}(X)$ can be represented by an element of the form $[E]-[\underline{M}]$, where $E$ is a $V$-bundle and $M$ is a $V$-module.

(2) If $[E]=[F]$ in $K_{V}(X)$ then there is a $V$-module $M$ such that $E \oplus \underline{M} \cong$ $F \oplus \underline{M}$.

Proof : As we have mentioned already, every element of $K_{V}(X)$ is of the form $[H]-[G]$. By Proposition 3.4, there exists a $V$-bundle $F$ and a $V$-module $M$ such that $H \oplus F \cong \underline{M}$. Thus we have

$$
[G]-[H]=[G+F]-[H+F]=[G+F]-[\underline{M}] .
$$

This proves (1).

If $[E]=[F]$, then there exists a $V$-bundle $G$ such that $E \oplus G \cong F \oplus G$. Let $H$ be a $V$-bundle such that $G \oplus H \cong \underline{M}$ for some $V$-module $M$. Then we have $E \oplus \underline{M} \cong F \oplus \underline{M}$. This proves (2).

Clearly, $K_{V}(p t)$ is just the the Grothendieck group of $V$-modules. From Proposition 3.6 we have the following proposition. 
Proposition 4.6. If $V$ is rational, then

$$
K_{V}(X)=K(X) \otimes_{\mathbb{Z}} K_{V}(p t) .
$$

Remark 4.7. If $V$ is rational and $\left\{M^{1}, \ldots, M^{p}\right\}$ is the set of irreducible inequivalent $V$-modules up to isomorphisms, then $K_{V}(p t)$ is isomorphic to the group $\mathbb{Z}^{p}$ with generators $\left[M^{1}\right], \ldots,\left[M^{p}\right]$. In particular, for $V$-modules $M$ and $N,[M]=[N]$ if and only if $M \cong N$ as $V$-modules.

4.2. Definition of $K_{V}(X, Y)$. We next define $K_{V}(X, Y)$ for a compact pair $(X, Y)$. Let $\mathcal{C}$ denote the category of compact spaces, $\mathcal{C}^{+}$the category of compact spaces with distinguished basepoint, and $\mathcal{C}^{2}$ the category of compact pairs. We define a functor $\mathcal{C}^{2} \rightarrow \mathcal{C}^{+}$, by sending a pair $(X, Y)$ to $X / Y$ with base point $Y / Y$ (if $Y=\emptyset$, the empty set, $X / Y$ is understood to be the disjoint union of $X$ with a point.). If $X \in \mathcal{C}$, we denote $(X, \emptyset) \in \mathcal{C}^{2}$ by $X^{+}$.

If $X$ is in $\mathcal{C}^{+}$, we define $\widetilde{K}_{V}(X)$ to be the kernel of the map $i^{*}: K_{V}(X) \rightarrow$ $K_{V}\left(x_{0}\right)$ where $i: x_{0} \rightarrow X$ is the inclusion of the basepoint. If $c: X \rightarrow x_{0}$ is the collapsing map then $c^{*}$ induces a splitting $K_{V}(X)=\widetilde{K}_{V}(X) \oplus K_{V}\left(x_{0}\right)$. This splitting is clearly natural for maps in $\mathcal{C}^{+}$. Thus $\widetilde{K}_{V}$ is a functor on $\mathcal{C}^{+}$. Also, it is clear that $K_{V}(X) \simeq \widetilde{K}_{V}\left(X^{+}\right)$. We define $K_{V}(X, Y)$ by $K_{V}(X, Y)=\widetilde{K}_{V}(X / Y)$. In particular $K(X, \emptyset) \simeq K_{V}(X)$. Since $\widetilde{K}_{V}$ is a functor on $\mathcal{C}^{+}$it follows that $K_{V}(X, Y)$ is a contravariant functor of $(X, Y)$ in $\mathcal{C}^{2}$.

We now introduce the smash product operator in $\mathcal{C}^{+}$, if $X, Y \in \mathcal{C}^{+}$, we put $X \wedge Y=X \times Y / X \vee Y$ where $X \vee Y=X \times\left\{y_{0}\right\} \cup\left\{x_{0}\right\} \times Y, x_{0}, y_{0}$ being the base-points of $X, Y$ respectively. For any three spaces $X, Y, Z \in \mathcal{C}^{+}$, we have a natural homeomorphism $X \wedge(Y \wedge Z) \simeq(X \wedge Y) \wedge Z$ and we shall identify these spaces by the homeomorphism.

Let $I$ denote the unit interval $[0,1]$ and let $\partial I=\{0\} \cup\{1\}$ be its boundary. We take $I / \partial I \in \mathcal{C}^{+}$as our standard model of the circle $S^{1}$. For $X \in \mathcal{C}^{+}$the space $S^{1} \wedge X \in \mathcal{C}^{+}$is called the reduced suspension of $X$, and often written as $S X$. The $n$-th iterated suspension $S S \cdots S X$ ( $n$ times) is naturally homeomorphic to $S^{n} \wedge X$ and is written briefly as $S^{n} X$.

Definition 4.8. For $n \leq 0$,

$$
\begin{aligned}
& \widetilde{K}_{V}^{-n}(X)=\widetilde{K}_{V}\left(S^{n} X\right) \text { for } X \in \mathcal{C}^{+}, \\
& K_{V}^{-n}(X, Y)=\widetilde{K}_{V}^{-n}(X / Y)=\widetilde{K}_{V}\left(S^{n}(X / Y)\right) \text { for }(X, Y) \in \mathcal{C}^{2}, \\
& K_{V}^{-n}(X)=K_{V}^{-n}(X, \emptyset)=\widetilde{K}_{V}\left(S^{n}\left(X^{+}\right)\right) \text {for } X \in \mathcal{C} .
\end{aligned}
$$

By proceeding further, we define the cone on $X$ by

$$
C X=I \times X /\{0\} \times X .
$$

Thus $C$ is a functor $C: \mathcal{C} \rightarrow \mathcal{C}^{+}$. We identify $X$ with the subspace $\{1\} \times X$ of $C X$. The space $C X / X=I \times X / \partial I \times X$ is called the unreduced suspension of $X$. 


\section{Cohomological properties of $K$}

In this section, we assume that $X$ is a finite $\mathrm{CW}$-complex and $Y \subset X$ is a CW sub-complex. This condition allows us to extend a trivialization of $V$-bundles on $Y$ to a neighborhood of $Y$ which is crucial in the proof of Lemma 5.1, Theorem 5.3. We will establish the basic cohomological properties of $K_{V}$ for these compact pairs $(X, Y)$.

Lemma 5.1. We have an exact sequence

$$
K_{V}(X, Y) \stackrel{j^{*}}{\rightarrow} K_{V}(X) \stackrel{i^{*}}{\rightarrow} K_{V}(Y)
$$

where $i: Y \rightarrow X$ and $j:(X, \emptyset) \rightarrow(X, Y)$ are the inclusions.

Proof : The composition $i^{*} \circ j^{*}$ is induced by the composition $j \circ i:(Y, \emptyset) \rightarrow$ $(X, Y)$ and so factor through the zero group. Thus $i^{*} \circ j^{*}=0$. Suppose now that $\xi \in \mathrm{Ker}^{*}$. We may represent $\xi$ in the form $[E]-[\underline{M}]$ where $E$ is a $V$-bundle over $X$ and $M$ is a $V$-module. Since $i^{*} \xi=0$, it follows that $\left.[E]\right|_{Y}=[\underline{M}]$ in $K_{V}(Y)$. This implies that there exists a $V$-module $N$ such that we have

$$
\left.(E \oplus \underline{N})\right|_{Y}=\underline{M} \oplus \underline{N} .
$$

Now as $Y$ is a CW sub-complex of $X$, there exists an open neighborhood $U$ of $Y$ in $X$ such that $Y$ is a strong deformation retract of $U$, i.e. there exists $f_{t}: U \rightarrow U(t \in[0,1])$ such that $f_{1}=\operatorname{Id}_{U},\left.f_{0}\right|_{Y}=\operatorname{Id}_{Y}$ and $f_{0}(U)=Y$. By Lemma 4.4, $(5.2),\left.(E \oplus \underline{N})\right|_{U}$ is trivial on $U$. This defines a bundle $E \oplus \underline{N} / \alpha$ on $X / Y$ and so an element

$$
\tau=[E \oplus \underline{N} / \alpha]-[\underline{M} \oplus \underline{N}] \in \widetilde{K}_{V}(X / Y)=K_{V}(X, Y) .
$$

Then

$$
j^{*}(\tau)=[E \oplus \underline{N} / \alpha]-[\underline{M} \oplus \underline{N}]=[E]-[\underline{M}]=\xi .
$$

Thus Ker $i^{*}=\operatorname{Im} j^{*}$ and the exactness is established.

Corollary 5.2. If $(X, Y) \in \mathcal{C}^{2}$ and $Y \in \mathcal{C}^{+}$, then the following sequence is exact,

$$
K_{V}(X, Y) \rightarrow \widetilde{K}_{V}(X) \rightarrow \widetilde{K}_{V}(Y)
$$

Proof : This is immediate from Lemma 5.1 and the natural isomorphisms

$$
\begin{aligned}
K_{V}(X) & \simeq \widetilde{K}_{V}(X) \oplus K_{V}\left(y_{0}\right), \\
K_{V}(Y) & \simeq \widetilde{K}_{V}(Y) \oplus K_{V}\left(y_{0}\right) .
\end{aligned}
$$

Our main proposition of this section is following,

Theorem 5.3. There is a natural exact sequence (infinite to the left)

$$
\begin{aligned}
& \cdots \rightarrow K_{V}^{-2}(Y) \stackrel{\delta}{\rightarrow} K_{V}^{-1}(X, Y) \stackrel{j^{*}}{\rightarrow} K_{V}^{-1}(X) \stackrel{i^{*}}{\rightarrow} K_{V}^{-1}(Y) \\
& \stackrel{\delta}{\rightarrow} K_{V}^{0}(X, Y) \stackrel{j^{*}}{\rightarrow} K_{V}^{0}(X) \stackrel{i^{*}}{\rightarrow} K_{V}^{0}(Y) .
\end{aligned}
$$


Proof : First we observe that it is sufficient to show that

$$
K_{V}^{-1}(X, Y) \stackrel{j^{*}}{\rightarrow} K_{V}^{-1}(X) \stackrel{i^{*}}{\rightarrow} K_{V}^{-1}(Y) \stackrel{\delta}{\rightarrow} \widetilde{K}_{V}^{0}(X, Y) \stackrel{j^{*}}{\rightarrow} \widetilde{K}_{V}^{0}(X) \stackrel{i^{*}}{\rightarrow} \widetilde{K}_{V}^{0}(Y)
$$

is exact. In fact, if this has been established then, by replacing $(X, Y)$ by $\left(S^{n} X, S^{n} Y\right)$ for $n=1,2, \cdots$, we obtain an infinite sequence continuing (5.8). Then by replacing $(X, Y)$ by $\left(X^{+}, Y^{+}\right)$where $(X, Y)$ is any pair in $\mathcal{C}^{2}$ we get the infinite sequence of the enunciation. Now, Corollary 5.2 gives the exactness of the last three terms of (5.8). To get exactness at the remaining places we shall apply Corollary 5.2 in turn to the pairs $(X \cup C Y, X)$ and $((X \cup C Y) \cup C X, X \cup C Y)$. First, by taking the pair $(X \cup C Y, X)$, we get an exact sequence

$$
K_{V}(X \cup C Y, X) \stackrel{\tau_{1}^{*}}{\rightarrow} \widetilde{K}_{V}(X \cup C Y) \stackrel{\tau_{2}^{*}}{\rightarrow} \widetilde{K}_{V}(X),
$$

where $\tau_{1}, \tau_{2}$ are the natural inclusions. Let $U$ be the neighborhood of $Y$ in $X$ as in Lemma 5.1. Since $C Y$ is contractible, by Lemma 4.4, any $V$-bundle $E$ on $X \cup C Y$ is trivial on $U \cup C Y$, thus $p^{*}: \widetilde{K}_{V}(X / Y) \rightarrow \widetilde{K}_{V}(X \cup C Y)$ is an isomorphism where $p: X \cup C Y \rightarrow X \cup C Y / C Y=X / Y$ is the collapsing map. Also the composition $\tau_{2}^{*} \circ p^{*}$ coincides with $j^{*}$. Let $\theta: K_{V}(X \cup C Y, X) \rightarrow K_{V}^{-1}(Y)$ be the isomorphism introduced earlier. Then by defining $\delta: K_{V}^{-1}(Y) \rightarrow K_{V}(X, Y)$ by $\delta=\tau_{1}^{*} \circ \theta^{-1}$, we obtain the exact sequence

$$
K_{V}^{-1}(Y) \stackrel{\delta}{\rightarrow} K_{V}(X, Y) \stackrel{j^{*}}{\rightarrow} \widetilde{K}_{V}(X)
$$

which is the middle part of (5.8).

Finally, we apply Corollary 5.2 to the pair $\left(X \cup C_{1} Y \cup C_{2} X, X \cup C_{1} Y\right)$, where we have labeled the cones $C_{1}$ and $C_{2}$ in order to distinguish between them. Thus we obtain an exact sequence

$$
K_{V}\left(X \cup C_{1} Y \cup C_{2} X, X \cup C_{1} Y\right) \rightarrow \widetilde{K}_{V}\left(X \cup C_{1} Y \cup C_{2} X\right) \rightarrow \widetilde{K}_{V}\left(X \cup C_{1} Y\right) .
$$

It will be sufficient to show that this sequence is isomorphic to the sequence obtained from the first three terms of (5.8). In view of the definition of $\delta$, it will be sufficient to show that the following diagram commutes up to sign.

$$
\begin{array}{rlrl}
K_{V}\left(X \cup C_{1} Y \cup C_{2} X, X \cup C_{1} Y\right) & =\widetilde{K}_{V}\left(C_{2} X / X\right)=K_{V}^{-1}(X) \\
\downarrow & & \widetilde{K}_{V}\left(C_{1} Y / Y\right)=K_{V}^{i^{*} \downarrow}(Y) .
\end{array}
$$

As in $[1, \mathrm{p} 74]$, we will get $(5.12)$ if we can prove that the following diagram commutes up to sign,

$$
\begin{aligned}
K_{V}\left(C_{1} Y \cup C_{2} Y\right) & \leftarrow K_{V}\left(C_{1} Y / Y\right) \leftarrow \widetilde{K}_{V}(S Y) \\
& \nwarrow K_{V}\left(C_{2} Y / Y\right) \leftarrow \widetilde{K}_{V}(S Y) .
\end{aligned}
$$

This follow from the following lemma 5.4. 
Lemma 5.4. Let $T: S^{1} \rightarrow S^{1}$ be defined by $T(t)=1-t, t \in I=[0,1]$. Recall that $S^{1}=I / \partial I$. Let $T \wedge 1: S Y \rightarrow S Y$ be the map induced by $T$ on $S^{1}$ and the identity on $Y$ for $Y \in \mathcal{C}^{+}$. Then $(T \wedge 1)^{*} a=-a$ for $a \in \widetilde{K}_{V}(S Y)$.

Proof : By the construction and Lemma 4.5, for any $a \in \widetilde{K}_{V}(S Y)$, there exist a $V$-module $M$ and a $V$-bundle $E$ on $S Y$ such that $a=[E]-[\underline{M}]$. We define $E$ first. As $S Y=C_{1} Y \cup_{Y} C_{2} Y$, and $C_{i} Y(i=1,2)$ is contractible, we know that $\left.E\right|_{C_{i} Y}$ are trivial, thus there are maps $f_{i}:\left.E\right|_{C_{i} Y} \rightarrow C_{i} Y \times M$ of $V$-module isomorphism. The composite $f=f_{2} \circ f_{1}^{-1}=\left(f^{\lambda}\right)_{\lambda \in \mathbb{C}}: Y \rightarrow \operatorname{Aut}_{V}(M)$ is well defined. Now, the operation $(T \wedge 1)^{*}$ on $[E]$ corresponds to the operation of replacing the map $y \rightarrow f(y)$ by $y \rightarrow f(y)^{-1}=\left(\left(f^{\lambda}\right)^{-1}\right)_{\lambda \in \mathbb{C}}$. We denote the corresponding bundle by $E_{1} \in K_{V}(S Y)$. We need to prove that in $K_{V}(S Y)$,

$$
[E] \oplus\left[E_{1}\right]=[\underline{M}] \oplus[\underline{M}] \text {. }
$$

For $0 \leq t \leq \pi / 2, y \in Y$, set

$$
F_{t}(y)=\left(\begin{array}{cc}
f(y) & 0 \\
0 & 1
\end{array}\right)\left(\begin{array}{cc}
\cos (t) & \sin (t) \\
-\sin (t) & \cos (t)
\end{array}\right)\left(\begin{array}{cc}
1 & 0 \\
0 & f(y)^{-1}
\end{array}\right)\left(\begin{array}{cc}
\cos (t) & -\sin (t) \\
\sin (t) & \cos (t)
\end{array}\right) .
$$

Then

$$
F_{\pi / 2}(y)=\left(\begin{array}{ll}
1 & 0 \\
0 & 1
\end{array}\right), \quad F_{0}(y)=\left(\begin{array}{cc}
f(y) & 0 \\
0 & f(y)^{-1}
\end{array}\right)
$$

This means that $F_{t}$ is a homotopy from $\left(\begin{array}{ll}1 & 0 \\ 0 & 1\end{array}\right)$ to $\left(\begin{array}{cc}f(y) & 0 \\ 0 & f(y)^{-1}\end{array}\right)$. Thus we get (5.14) from Lemma 3.3.

Now, by Lemma 4.4 and Theorem 5.3, we get

Corollary 5.5. If $Y$ is a retract of $X$, then for all $n \leq 0$, the sequence

$$
K_{V}^{-n}(X, Y) \rightarrow K_{V}^{-n}(X) \rightarrow K_{V}^{-n}(Y)
$$

is a split short exact sequence, and

$$
K_{V}^{-n}(X) \simeq K_{V}^{-n}(X, Y) \oplus K_{V}^{-n}(Y) .
$$

\section{Associative algebra bundles}

This section is motivated by the relation between a vertex operator algebra $V$ and its Zhu's algebra $A(V)$. It turns out we can define associative algebra bundles for a large class of associative algebras.

6.1. Definition of associative algebra bundles. We assume that $A$ is an associative algebra over $\mathbb{C}$ with an anti-involution $\sigma$. The setting and results in Sections 2-5 hold in the present situation with suitable modifications.

Let $M$ be an $A$-module and we denote the dual space of $M$ by $M^{\prime}$ as before. We also denote the natural pairing $M^{\prime} \times M \rightarrow \mathbb{C}$ by $\left(m^{\prime}, m\right)$ for $m^{\prime} \in M^{\prime}$ and $m \in M$. The following lemma is obvious. 
Lemma 6.1. $M^{\prime}$ is also a A-module such that $\left(a m^{\prime}, m\right)=\left(m^{\prime}, \sigma(a) m\right)$ for $a \in$ $A, m^{\prime} \in M^{\prime}$ and $m \in M$.

As in Section 2 a form ( , ) on an $A$-module $W$ is called invariant if $\left(a w_{1}, w_{2}\right)=$ $\left(w_{1}, \sigma(a) w_{2}\right)$ for $w_{i} \in W$ and $a \in A$.

Lemma 6.2. Lemma 2.8 holds for an A-module $M$.

We also need to define the category $\mathcal{O}_{A}$ of $A$-modules. An $A$-module $W$ is in $\mathcal{O}_{A}$ if there exist $\lambda_{1}, \ldots, \lambda_{s} \in \mathbb{C}$ such that $W=\bigoplus_{i=1}^{s} \bigoplus_{n>0} W_{\lambda_{i}+n}$ is a direct sum of finite dimensional $A$-modules and such that $\operatorname{Hom}_{A}\left(W_{\lambda}, W_{\mu}\right)=0$ if $\mu \neq \lambda$. Such definition of category $\mathcal{O}_{A}$ is well justified by Remark 6.4, Theorems 6.7 (7) and 6.8 below.

Definition 6.3. Let $X$ be a compact space and $W \in \mathcal{O}_{A}$. An $A$-bundle $E$ over $X$ with fiber $W$ is a direct sum of vector bundles $E=\oplus_{\lambda \in \mathbb{C}} E^{\lambda}$ over $X$ such that all transition functions are $A$-module isomorphisms. That is, there exists an open covering $\left\{X_{\alpha}\right\}_{\alpha \in I}$ of $X$ and a family of continuous isomorphisms of vector bundles $H_{\alpha}=\left(H_{\alpha}^{\lambda}:\left.E^{\lambda}\right|_{X_{\alpha}} \rightarrow X_{\alpha} \times M_{\lambda}\right)_{\lambda \in \mathbb{C}}$ with $M=\oplus_{\lambda \in \mathbb{C}} M_{\lambda}$ an $A$-module such that if we denote by $\left(H_{\alpha}^{\lambda} \circ\left(H_{\beta}^{\lambda}\right)^{-1}\right)_{\lambda \in \mathbb{C}}=\left(g_{\alpha \beta}^{\lambda}\right)_{\lambda \in \mathbb{C}}$, then each $g_{\alpha \beta}^{\lambda}(x): M_{\lambda} \rightarrow M_{\lambda}$ is an $A$-module isomorphism for any $x \in X_{\alpha} \cap X_{\beta}, \lambda \in \mathbb{C}$. In particular, each $E^{\lambda}$ is an $A$-bundle.

The analogues of Remark 3.2 are as follows:

Remark 6.4. (1) If $A=\mathbb{C}$, then $\sigma$ is necessarily the identity map, the $A$-bundle defined here is exactly the classical complex vector bundle over $X$.

(2) Let $A$ and $B$ be two associative algebras with anti-involutions $\sigma_{A}$ and $\sigma_{B}$ respectively. Then $A \otimes_{\mathbb{C}} B$ is an associative algebra with anti-involution $\sigma_{A} \otimes \sigma_{B}$. Assume $E, F$ are the $A$ and $B$-bundles over $X$, then $E \otimes F$ is a $A \otimes_{\mathbb{C}} B$-bundle over $X$. In particular, If $A=\mathbb{C}$ then $E \otimes F$ again is a $B$-bundle over $X$.

We can also define subbundles, quotient bundles, direct sum of bundles. Various bundle homomorphisms are also defined as expected.

Let $E$ be an $A$-bundle over $X$. Regarding $E$ as a vector bundle over $X$, the dual bundle $E^{\prime}$ is also an $A$-bundle.

Lemma 3.3 and Proposition 3.4 hold with $V$ replaced by $A$.

We also define the $K$-group $K_{A}(X)$ to be the abelian group generated by the equivalence classes of $A$-bundles. Then Lemmas $4.2,4.4,4.5$ and Corollary 4.3 hold with obvious changes. Proposition 4.6 is also true if $A$ is semisimple (that is, $A$ is a direct sum of full matrix algebras) and with $V$ replaced by $A$. That is $K_{A}(X)=K(X) \otimes_{\mathbb{Z}} K_{A}(p t)$.

We can also define $K_{A}(X, Y)$ and related objects as in Section 4 and the cohomological properties in Section 5 also hold in the present setting.

6.2. Zhu's algebra $A(V)$. We review the Zhu's algebra $A(V)$ in this subsection following [15] and [5]. 
Let $V$ be a vertex operator algebra. For any homogeneous vectors $a \in V$, and $b \in V$, we define

$$
\begin{aligned}
& a * b=\left(\operatorname{Res}_{z} \frac{(1+z)^{\mathrm{wt} a}}{z} Y(a, z)\right) b, \\
& a \circ b=\left(\operatorname{Res}_{z} \frac{(1+z)^{\mathrm{wt} a}}{z^{2}} Y(a, z)\right) b,
\end{aligned}
$$

and extend to $V \times V$ bilinearly, here $\operatorname{Res}_{z}$ denotes the coefficient of $z^{-1}$. Denote by $O(V)$ the linear span of $a \circ b(a, b \in V)$ and set $A(V)=V / O(V)$. We write $[a]$ for the image $a+O(V)$ of $a \in V$.

For homogeneous $a \in V$ we set $o(a)=a_{\mathrm{wt} a-1}$ and extend linearly to all $a \in V$. If $M=\bigoplus_{n>0} M(n)$ is an admissible module then $o(a) M(n) \subset M(n)$ for all $a \in V$ and $n \in \overline{\mathbb{Z}}$. We now define the space of lowest weight vectors of $M$ :

$$
\Omega(M)=\left\{w \in M \mid a_{\mathrm{wt} a+m} w=0, a \in V, m \geq 0\right\} .
$$

Remark 6.5. If $M=\bigoplus_{\lambda \in \mathbb{C}} M_{\lambda}$ is a $V$-module then $\Omega(M)=\bigoplus_{\lambda \in \mathbb{C}} \Omega(M)_{\lambda}$ is naturally graded and each homogeneous subspace $\Omega(M)_{\lambda}=\Omega(M) \cap M_{\lambda}$ is finite dimensional.

The following lemma is evident.

Lemma 6.6. If $M$ and $W$ are admissible $V$-modules and $f: M \rightarrow W$ is a $V$-module homomorphism then $f(\Omega(M)) \subset \Omega(W)$. In particular, if $f$ is an isomorphism then $f(\Omega(M))=\Omega(W)$.

The following theorem is due to $[15, \S 2]$ (also see [5]).

Theorem 6.7. (1) The bilinear operation * induces on $A(V)$ an associative algebra structure. The vector $[\mathbf{1}]$ is the identity and $[\omega]$ is in the center of $A(V)$.

(2) The linear map

$$
\phi: a \mapsto e^{L(1)}(-1)^{L(0)} a
$$

induces an anti-involution of $A(V)$.

(3) Let $M=\bigoplus_{n=0}^{\infty} M(n)$ be an admissible $V$-module with $M(0) \neq 0$. Then the linear map

$$
o: V \rightarrow \operatorname{End}(\Omega(M)), a \mapsto o(a) \mid \Omega(M)
$$

induces an algebra homomorphism from $A(V)$ to $\operatorname{End}(\Omega(M))$. Thus $\Omega(M)$ is a left $A(V)$-module.

(4) The map $M \mapsto M(0)$ induces a bijection from the set of equivalence classes of irreducible admissible $V$-modules to the set of equivalence classes of irreducible $A(V)$-modules.

(5) If $M=\bigoplus_{\lambda \in \mathbb{C}} M_{\lambda}$ is a $V$-module, then each $\Omega(M)_{\lambda}$ is an finite-dimensional $A(V)$-module.

(6) If $V$ is rational then $A(V)$ is a finite dimensional semisimple algebra. Moreover, $M \rightarrow \Omega(M)$ gives an equivalence of the category of admissible $V$ modules and the category of $A(V)$-modules and the same functor also gives an 
equivalence of the category of $V$-modules and the category of finite dimensional $A(V)$-modules.

(7) If $M \in \mathcal{O}_{V}$ then $\Omega(M) \in \mathcal{O}_{A(V)}$.

Note that if $M$ is a $V$-module and $\lambda \neq \mu$ then $\operatorname{Hom}_{A(V)}\left(\Omega(M)_{\lambda}, \Omega(M)_{\mu}\right)=0$ as $[\omega]$ acts on $\Omega(M)_{\mu}$ as scalar $\mu$. Thus $\Omega(M)$ is an element of $\mathcal{O}_{A(V)}$. In fact, the definition of $\mathcal{O}_{A}$ for an associative algebra $A$ reflects well the properties of $\Omega(M)$.

6.3. Relationship between $K_{V}(X)$ and $K_{A(V)}(X)$. The relationship between $K_{V}(X)$ and $K_{A(V)}(X)$ is a reflection of the relation between $V$ and $A(V)$ for any vertex operator algebra $V$ and compact space $X$.

Recall Definition 3.1. Let $E$ be a $V$-bundle over $X$ with fiber $M=\bigoplus_{\lambda \in \mathbb{C}} M_{\lambda}$. Let $\left\{X_{\alpha} \mid \alpha \in I\right\}$ be an open covering of $X$ which gives a local trivialization of $E$. We define a graded vector bundle $\Omega(E)=\bigoplus_{\lambda \in \mathbb{C}} \Omega(E)^{\lambda}$ in the following way: set $\left.\Omega(E)^{\lambda}\right|_{X_{\alpha}}=\left(H_{\alpha}^{\lambda}\right)^{-1}\left(X_{\alpha} \times \Omega(M)_{\lambda}\right)$ and $\Omega\left(H_{\alpha}\right)^{\lambda}=\left.H_{\alpha}\right|_{\Omega(E)^{\lambda}}$. Then $\Omega\left(H_{\alpha}\right)^{\lambda}$ : $\left.\Omega(E)^{\lambda}\right|_{X_{\alpha}} \rightarrow X_{\alpha} \times \Omega(M)_{\lambda}$ is an isomorphism. Set $\Omega\left(H_{\alpha}\right)=\left(\Omega\left(H_{\alpha}\right)^{\lambda}\right)_{\lambda \in \mathbb{C}}$ and $\Omega\left(g_{\alpha \beta}\right)^{\lambda}=\Omega\left(H_{\alpha}\right)^{\lambda} \circ\left(\Omega\left(H_{\beta}\right)^{\lambda}\right)^{-1}$ for $\lambda \in \mathbb{C}$.

Then $\Omega\left(g_{\alpha \beta}\right)(x)=\left(\Omega\left(g_{\alpha \beta}\right)^{\lambda}(x)\right)_{\lambda \in \mathbb{C}}: \Omega(M) \rightarrow \Omega(M)$ is an $A(V)$-module isomorphism for any $x \in X_{\alpha} \cap X_{\beta}$ by Lemma 6.6. By Theorem $6.7(7), \Omega(E)$ is an $A(V)$-bundle over $X$.

Theorem 6.8. The map $\Omega: E \rightarrow \Omega(E)$ from the set of $V$-bundles over $X$ to the set of $A(V)$-bundles over $X$ induces a homomorphism, which we still denote by $\Omega$, from the group $K_{V}(X)$ to the group $K_{A(V)}(X)$. In particular, if $V$ is rational, $\Omega$ is an isomorphism.

Proof : It is clear that $\Omega$ is a group homomorphism. Now we assume that $V$ is rational. Recall Proposition 4.6 and its analogue $K_{A(V)}(X)=K(X) \otimes_{\mathbb{Z}}$ $K_{A(V)}(p t)$ for $A(V)$. Using Theorem 6.7 (5) we need to prove that $K_{V}(p t)$ and $K_{A(V)}(p t)$ are isomorphic groups under the map $\Omega$. But this is clear from Theorem $6.7(5)$ again by noting that the category $\mathcal{O}_{A(V)}$ is exactly the category of finite dimensional $A(V)$-modules.

\section{References}

[1] M.F. Atiyah, K-theory. W. A. Benjamin, Inc., New York-Amsterdam 1967

[2] R. Borcherds, Vertex algebras, Kac-Moody algebras, and the Monster, Proc. Natl. Acad. Sci. USA 83 (1986), 3068-3071.

[3] C. Dong and J. Lepowsky, Generalized Vertex Algebras and Relative Vertex Operators, Progress in Math. 112, Birkhäuser, Boston 1993.

[4] C. Dong, H. Li and G. Mason, Regularity of rational vertex operator algebras, Adv. in Math. 132 (1997), 148-166.

[5] — , Twisted representations of vertex operator algebras, Math. Ann. 310 (1998), 571-600.

[6] C. Dong and G. Mason, Vertex operator algebras and their automorphism groups, Representations and quantizations (Shanghai, 1998), 145-166, China High. Educ. Press, Beijing, 2000 
[7] E. Frenkel and D. Ben-Zvi, Vertex algebras and algebraic curves. Mathematical Surveys and Monographs, 88. AMS, 2001.

[8] I.B. Frenkel, Y. Huang and J. Lepowsky, On axiomatic approach to vertex operator algebras and modules, Mem. Amer. Math. Soc. 104, 1993.

[9] I.B. Frenkel, J. Lepowsky and A. Meurman, Vertex operator algebras and the Monster, Academic Press, 1988.

[10] I.B. Frenkel and Y. Zhu, Vertex operator algebras associated to representations of affine and Virasoro algebras. Duke Math. J. 66 (1992), 123-168.

[11] A. Hatcher, Vector bundles and K-Theory, Cornell Lecture Notes.

[12] V. Kac, Infinite-dimensional Lie algebras, Cambridge Univ. Press, London, 1991.

[13] H.Li, Local systems of vertex operators, vertex superalgebras and modules, J. Pure Appl. Algebra 109 (1996), 143-195.

[14] E. Witten, The index of the Dirac operator in loop space, Elliptic Curves and Modular forms in Algebraic Topology, Landweber P.S., SLNM 1326, Springer, Berlin, 161-186.

[15] Y. Zhu, Modular invariance of characters of vertex operator algebras, J. AMS 9, (1996), $237-301$.

Mathematics Department, University of California, Santa Cruz, CA 95064, U.S.A. E-mail address: dong@math.ucsc.edu

Center of Mathematical Sciences, Zhejiang University and Department of MathEMATICS, UCLA,CA 90095-1555, USA

E-mail address: liu@math.ucla.edu

Centre de Mathématiques, UMR 7640 du CNRS, Ecole Polytechnique, 91128

Palaiseau Cedex, France

E-mail address: ma@math.polytechnique.fr

Department of Mathematics, Qinghua University, Beijing, China

E-mail address: jzhou@math.tsinghua.edu.cn 